\title{
A new approach for an ultra-thin piezoresistive sensor based on solidified carbon ink film
}

\author{
Ying $\mathrm{Yi}^{1}$ (D), Ayman Samara ${ }^{2}$, and Bo $\mathrm{Wang}^{1, *}$ (D) \\ ${ }^{1}$ Division of Information and Computing Technology, College of Science and Engineering, Hamad Bin Khalifa University, \\ Doha 34110, Qatar \\ ${ }^{2}$ Qatar Environment and Energy Research Institute, Hamad Bin Khalifa University, Doha 34110, Qatar
}

Received: 13 July 2020

Accepted: 5 September 2020

Published online:

29 September 2020

(C) The Author(s) 2020

\begin{abstract}
Conventional flexible piezoresistive strain sensors that use conductive particles polymer composites exhibit thick structures with a low sensitivity to external tension. This paper presents a cost-effective method to fabricate ultra-thin and highly sensitive piezoresistive strain sensors. In our fabrication steps, carbon ink that is mainly composed of carbon black particles is solidified with a drying process to form a "paperlike," flexible conductive film. Without any surface modification techniques, the carbon ink film is directly placed onto liquid-state PDMS and then bonded after the drying process. Following the rapid prototyping, different performance metrics of the fabricated sensors, including piezoresistivity, gauge factor, temperature dependency, elastic modulus, and repeatability are measured. Specifically, sensors fabricated with this method show a significantly improved gauge factor ( 26$)$ compared to similar flexible sensors fabricated by more complicated micro-fabrication methods. The proposed method of fabrication and the corresponding ultra-thin $(\sim 45 \mu \mathrm{m})$ sensor prototype may benefit the design and mass production of future wearable biomedical and healthcare sensors.
\end{abstract}

\section{Introduction}

Flexible and lightweight strain sensors are critical components for applications such as human motion detection [1, 2], touch panels [3, 4], soft robotics [5], electronic skin [6, 7], wearable electronics [8], health monitoring $[9,10]$. Typically, a strain sensor exhibits piezoelectric [11, 12], capacitive [13, 14], or piezoresistive [15-17] transduction to external forces. While piezoelectric materials, such as $\mathrm{P}(\mathrm{VDF}-\mathrm{TrFE})$, produce electrical charges when mechanical stress is applied [11], the materials are usually stiff [18] and only able to function in conditions of dynamic tension [11], which greatly limits their applications. Capacitive strain sensors are made of 2 parallel

Handling Editor: Maude Jimenez.

Address correspondence to E-mail: bwang@hbku.edu.qa 
conductive electrodes with a dielectric layer between the electrodes. When an external force is applied, the property of the dielectric layer changes, which in turn, leads to a change in capacitance. The sensitivity of the capacitive strain sensor, however, is low when the effective compression applied to the dielectric layer is weak (e.g., a capacitance change ratio of $0.9 \%$ at a pulse pressure of $90 \mathrm{kPa}$ [19]). Therefore, piezoelectric and capacitive strain sensors face a challenge to achieve reliable measurements in conditions of static tension and/or weak strain, as in biomedical sensing applications [8]. In contrast to piezoelectric and capacitive sensors, piezoresistive sensors react to external tension with resistance changes. They can be designed using simple structures and are compatible with many read-out approaches [8]. Meanwhile, they provide high pressure sensitivity and quick response without much energy consumption [16]. Various materials, including copper nanowire [20], silver nanowire [21], gold nanowire [2], carbon nanotube (CNT) [22, 23], polymer nano-fibers [24, 25], metal particles [26, 27], graphene [28, 29], and carbon black $[30,31]$ can be used to fabricate piezoresistive sensors.

Although many piezoresistive strain sensors have been used in engineering practice, the design, material, and structure still have room for improvement. For example, a tactile sensor may have a strict thickness requirement $(<0.13 \mathrm{~mm})$ for pressure monitoring [31], and most of the reported sensors have not taken this into account [27, 32, 33]. Moreover, for wearable sensing applications, the sensor must be soft and lightweight and conform to irregular surfaces and must be flexible enough to comply with bodily motion. Therefore, the selection of the elastomeric substrate is critical. Polydimethylsiloxane (PDMS) has been widely used as the substrate for various strain sensors due to its flexibility, biocompatibility, chemical inertness, and stretchability $[32,33]$. Nevertheless, PDMS shows poor adhesion to many sensing materials and therefore, increases the risk of delamination. To properly integrate the sensing material with the PDMS substrate, micro-structures such as the graphene porous network structure [34], carbon nanotube-polymer sponge [35, 36], micro-electro-mechanical system (MEMS) [37], and the bionic hierarchical structure [38] have been proposed; however, these structures require complicated fabrication procedures and long processing times (more than $12 \mathrm{~h} \mathrm{[39]).}$
High sensitivity, light weight, cost-efficiency, excellent flexibility, rapid prototyping, and thin structure are highly desirable properties for wearable strain sensors, especially in biomedical and healthcare-related applications [40]. For example, an ultrathin PDMS membrane-based piezoresistive sensor can be used in diaphragm-actuated drug delivery devices to monitor the dosage [41, 42]. In this work, we design and fabricate an ultra-thin flexible piezoresistive sensor with high sensitivity using a low-cost fabrication scheme. In our approach, the carbon ink [43] is solidified and then directly placed onto the liquid-state PDMS, forming a robust piezoresistive sensor after the drying process. The fabrication time is much shorter than that of conventional approaches where carbon black particles are dispensed into liquid-state PDMS (e.g., more than $60 \mathrm{~h}$ of material preparation has been reported [44]). When the PDMS substrate is stretched, the sensor provides a resistance change due to the changing effective conductive path in the carbon ink [44, 45]. Moreover, to the best of our knowledge, the sensor in this work is the thinnest $(\sim 45 \mu \mathrm{m})$ of those reported in the literature. The sensor also provides a much higher gauge factor of 26, compared to prior articles that integrate PDMS substrate with CNT thin film [46], silver nanowire [47], or carbon black [30].

\section{Design and principle}

\section{Materials and fabrication}

The carbon ink used as the sensing material in this work is a colloid-state mixture of carbon black and ethylene glycol ( $\sim 18 \mathrm{wt} \%)$. Carbon black particles were uniformly distributed in the ethylene glycol to exhibit conductivity, and the resulting solution had a viscosity of $\sim 22 \mathrm{cP}[15,43]$. The carbon ink has little tendency to evaporate due to the high boiling temperature of ethylene glycol $\left(197^{\circ} \mathrm{C}\right)$. Figure 1 shows the fabrication steps of the PDMS substrate integrating the carbon ink film, which acts as the sensor. First, the polyacrylic acid (PAA) powder $\left(M_{\mathrm{w}}=1800\right.$, Sigma-Aldrich) was dissolved in distilled water at a $25 \% \mathrm{w} / \mathrm{v}$ concentration. The PAA solution was then filtered through a sterile $0.45 \mu \mathrm{m}$ PVDF syringe filter (Millipore Corporation, USA). Second, the PAA solution was coated onto 2 slides of glass with a spinning speed of $800 \mathrm{rpm}$ for $30 \mathrm{~s}$. The PAA-coated 


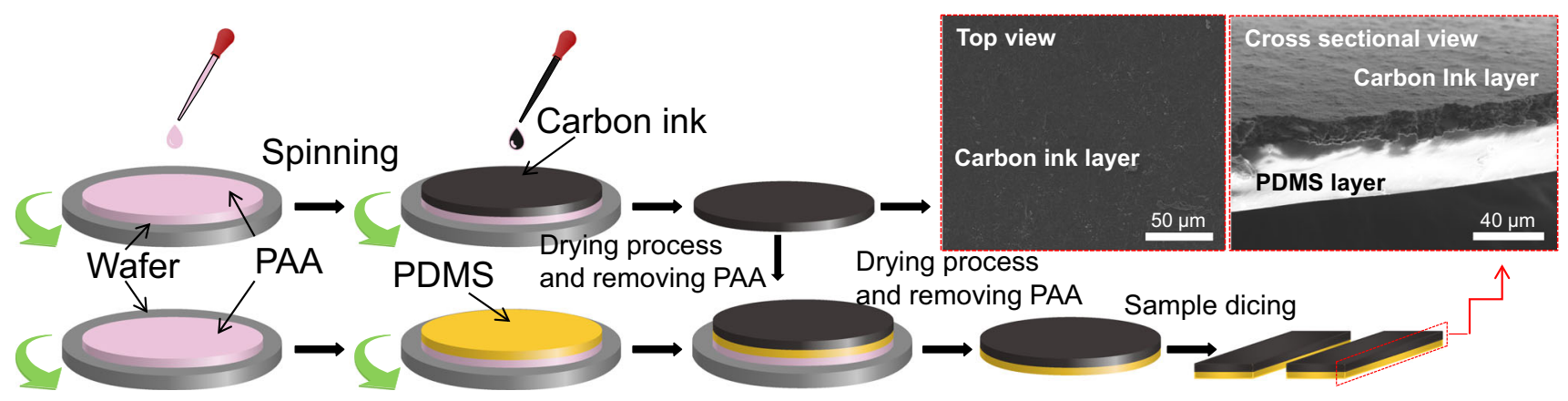

Figure 1 Fabrication steps for piezoresistive sensor showing the direct contact of the thin carbon ink layer with the PDMS substrate.

glass slides were pre-baked $\left(150{ }^{\circ} \mathrm{C}\right)$ for 5 min to dry the PAA, which would be used as the sacrificial layer. Next, the carbon ink was dropped onto one PAA coated glass and spun at $500 \mathrm{rpm}$ for $20 \mathrm{~s}$ and then at $1000 \mathrm{rpm}$ for $3 \mathrm{~min}$. The carbon ink was dried at $120{ }^{\circ} \mathrm{C}$ for $10 \mathrm{~min}$, and it was automatically peeled off from the glass after immersing the glass into water for a minimum of $5 \mathrm{~h}$.

The SEM image of the surface of the solidified carbon ink is shown in the insert of Fig. 1. A similar spinning operation was repeated to coat PDMS onto another PAA-coated glass slide. This glass was placed on a hot-plate after the carbon ink film had been evenly coated onto the liquid-state PDMS. After the drying process, the PDMS substrate was bonded to the carbon ink film. Lastly, the glass slide was immersed into water again leaving only the solidified PDMS and carbon ink, which was then diced and tailored into the sensor samples. The cross-sectional SEM image of the fabricated sensor samples shows $12.6 \mu \mathrm{m}$ of carbon ink film and $32.5 \mu \mathrm{m}$ of PDMS substrate in thickness. Although surface modifications of the solidified PDMS may promote its adhesion, as with a synthesized polyurethane urea (PUU) coating [48] or sodium dodecyl sulfate (SDS) treatments [49], in our fabrication steps, the liquid-state PDMS, as a relatively simple process, increases the adhesion between the functional sensing material and the polymer substrate [33].

\section{Working principle}

Differing from conventional strain sensors that use structures mixing conductive particles with polymer [44-47], the solidified carbon ink film is actually formed as a tight connection of carbon black particles that shows piezoresistivity. Its change in resistance is caused by the changes in conductive paths due to the:
(1) elongation of the sensor according to the common resistance calculation $R=\rho^{*}(L / A)$, where $\rho, L$, and $A$ denote material resistivity, length, and cross-sectional area of the conduct, respectively; (2) changes in the inner carbon black particle structured conductive link [45]. Figure 2 illustrates the process and principle. When an external tension is horizontally applied to the PDMS substrate, the carbon ink is elongated together with the substrate (Fig. 2a, b). As a result, the resistance change ratio $(\Delta R / R)$ of the sensor increases, where $\Delta R$ is the absolute resistance change and $R$ is the baseline resistance value of the sensor.

If, however, the substrate is over-stretched, the carbon ink will break, causing micron-scale cracks in the surface (Fig. 2c). This leads to permanently disconnected regions in the conductive link, and a sharp change in the $\Delta R / R$. Although the PDMS substrate is elastic and can be restored to its initial state when the tension is removed, the substrate may be too soft to completely heal the cracks (Fig. 2d). Consequently, the resistance of the sensor does easily revert to its original value, and a hysteresis phenomenon will likely occur (Fig. 2e). To prevent cracks and irreversible damage from occurring in the conductive path, the stretching of the sensor must be kept within safe limits.

\section{Experimental results of the fabricated sensor}

Following the fabrication procedure mentioned above, the sensor sample was tested under different levels of strain. The 2 ends of the sample were clamped and stretched through a dynamic mechanical analyzer (DMA Q800, TA Instruments, New Castle, DE, USA), with a corresponding experimental setup as depicted in Fig. 3a. The drive-shaft moved 
Figure 2 Solidified carbon ink film during over-stretched and recovering stages; SEM images of the surface. (a)

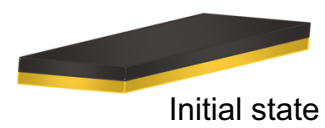

(b)

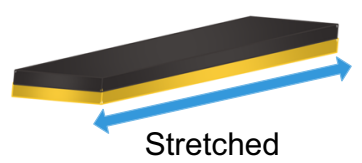

(e) (c)

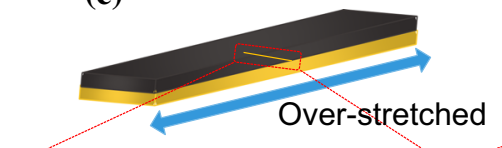

(d)
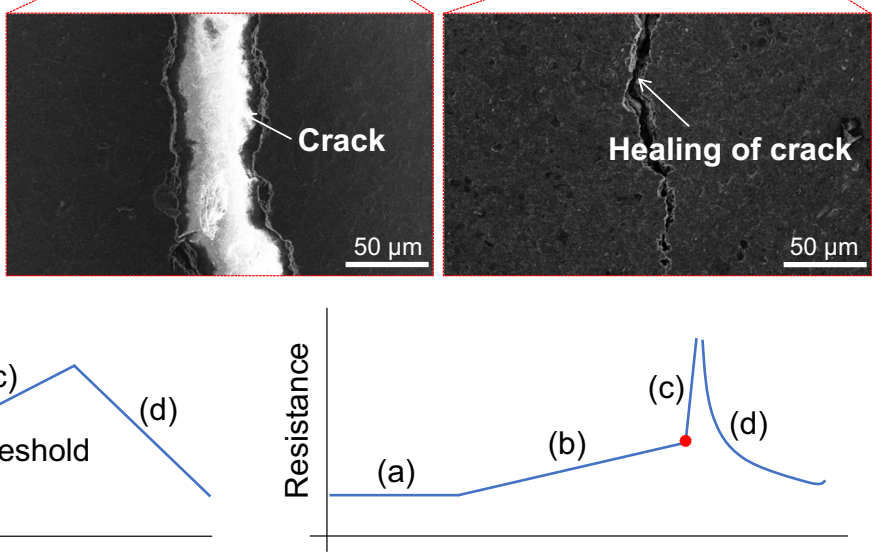

downwards, stretching the sensor sample to apply a specific strain value and it then reverted to its original position. A digital multimeter (GDM-8351, GW Instek Corp., New Taipei City, Taiwan) was used to record the resistance change of the sensor during the stretching process in real-time. The stretching operation was repeated 5-times for each strain value, and the average $\Delta R / R$ s were recorded (Fig. 3b). The fabricated sensor exhibited a linear increase in $\Delta R /$ $R$ with strains up to $5 \%$, and a maximum $\Delta R / R$ of $122.2 \pm 4.1 \%$ was achieved. In this test, no permanent delaminations or cracks in the carbon ink were observed, implying that a strain of $5 \%$ was within the safe stretching limits. Within the measurement range, dividing the average $\Delta R / R$ by the strain yielded a gauge factor of $25.5 \pm 0.9$ for the sensor. Clearly, our sensor shows a higher gauge factor than that reported by others using the method of mixing carbon black particles with PDMS [30]. This can be explained by the resistance change of the sensor that uses the mixing approach is derived only from changes in the inner effective conductive path. This requires a large strain to achieve a significant resistance change.

To design a flexible sensor for wearable or biomedical electronic applications, the temperature dependence and elastic module of the sensor were investigated in the body temperature range. The DMA was used to characterize the fabricated sensor in terms of temperature coefficient and elasticity. The sensor was placed in the furnace of the DMA, and its
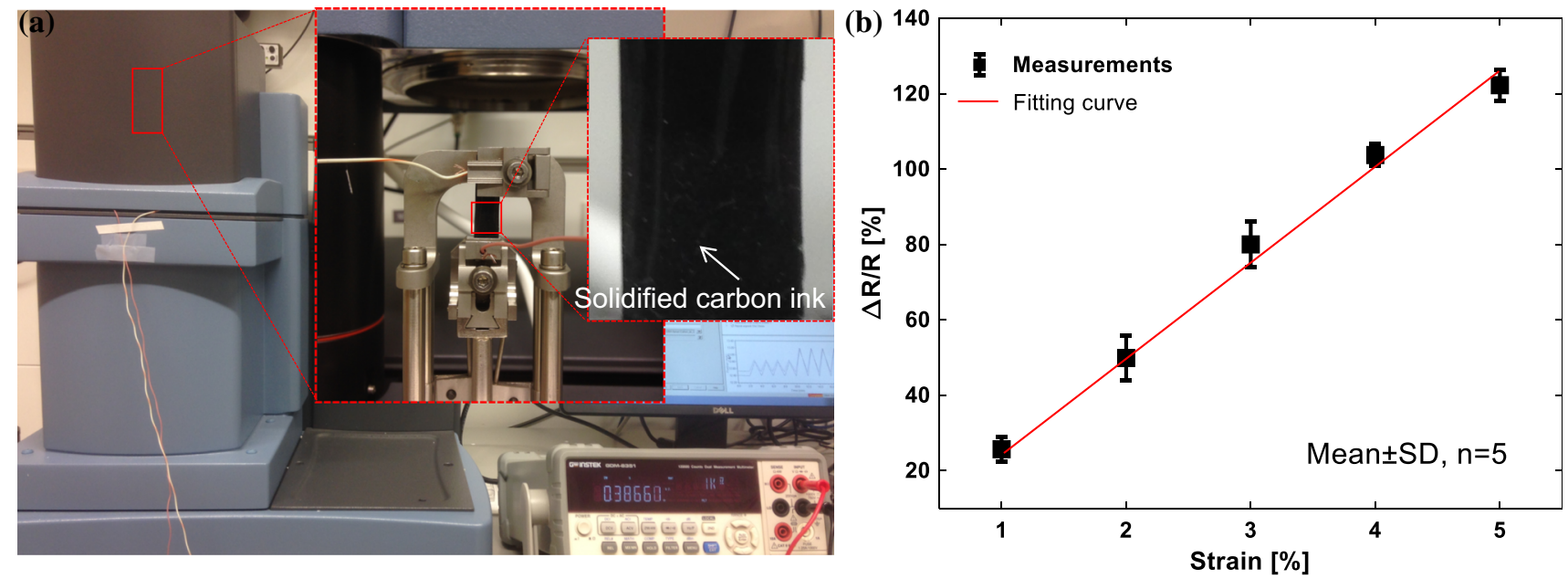

Figure 3 Experimental setup using a stretching model of the DMA and $\mathbf{b}$ measurements of $\Delta R / R$ corresponding to different levels of tensile strain in the stretching process. 
resistance (without tension) was measured at different temperatures. Figure 4 shows that temperature increases cause a linear resistance change, and the corresponding average $\Delta R / R(R=$ initial resistance at room temperature) of the 3 measurements increased to $1.4 \%$ at $37.5{ }^{\circ} \mathrm{C}$. Within the temperature range of $22.5-37.5{ }^{\circ} \mathrm{C}$, an average temperature coefficient of resistance $(\Delta R / \Delta T)$ of $0.37 \Omega /{ }^{\circ} \mathrm{C}$ was obtained. The elasticity of the sensor is also affected by temperature. Figure 5 shows that Young's modulus (the gradient of the stress-strain curve) for the sensor is doubled from 23 to $37^{\circ} \mathrm{C}$, implying that the elasticity of the sensor changes according to the sensor's location on the body since temperature is different at different locations. This feature should be considered for actual wearable electronic applications, and calibration techniques may need to be integrated with a given sensor for the purpose of obtaining accurate readings.

In the following experiments, the stability and repeatability of the sensor were evaluated. First, the DMA was used to stretch the sensor sample by ramping up the strain in steps of $1 \%$ for $3 \mathrm{~min}$, and statically holding the device for $1.5 \mathrm{~min}$ at each step, until the strain reached a maximum of 5\%. Figure 6 shows the $\Delta R / R$ of the sensor during the stretching and holding operations. The calculated $\Delta R / R$ was found to increase uniformly and a $\Delta R / R$ increase of $23 \pm 2.7 \%$ was obtained for each step. During the holding periods, the resistance of the sensor was maintained at a stable level even though a drift in $\Delta R / R$ of about $2.3 \%$ was observed due to the

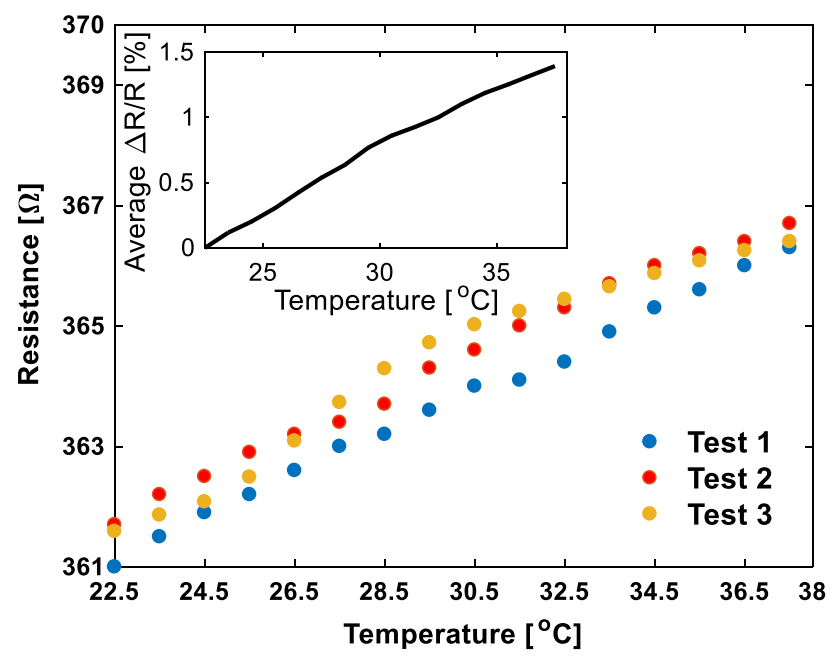

Figure 4 Temperature dependence of the sensor in a temperature range up to $37.5^{\circ} \mathrm{C}$.

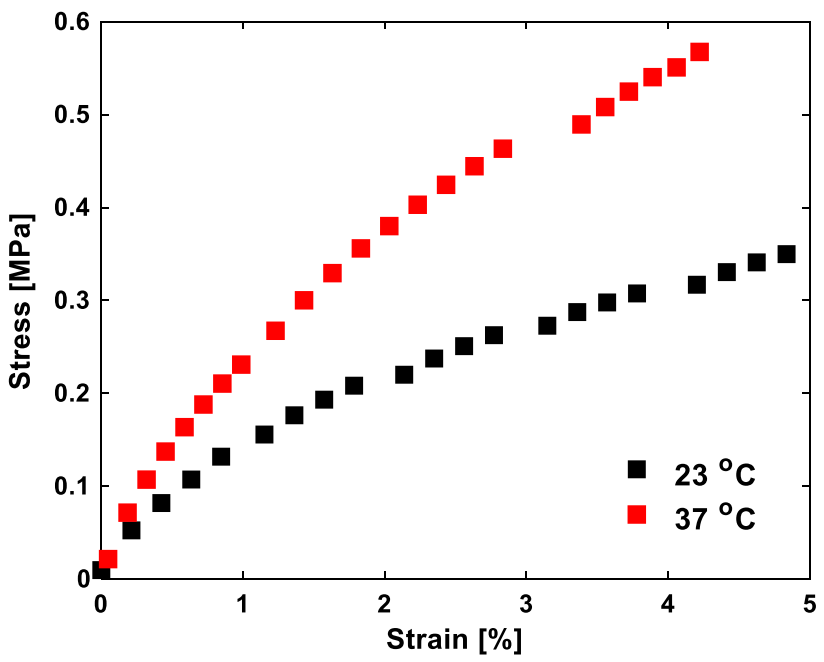

Figure 5 Young module of the sensor at different temperatures.

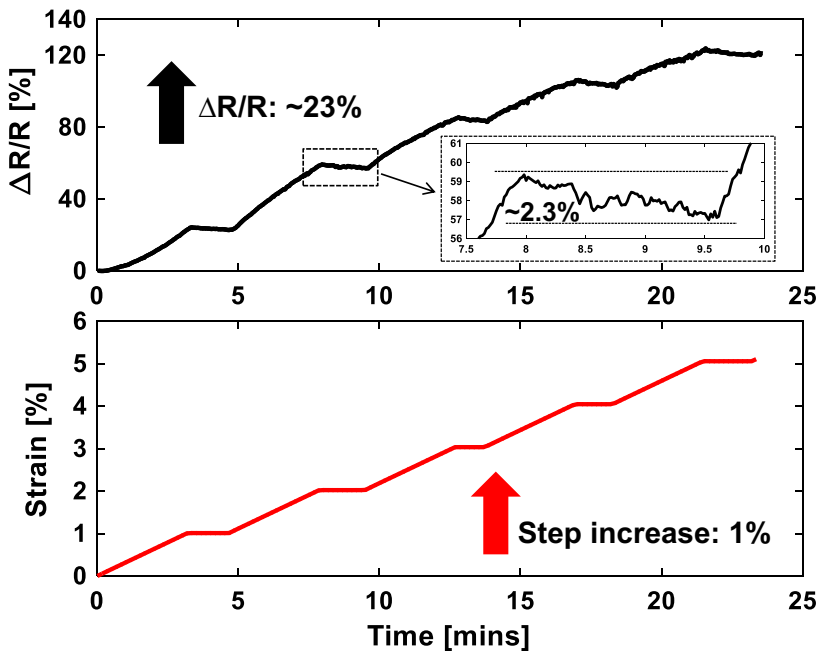

Figure $6 \Delta R / R$ of the sensor at different levels of strain and static tension.

stretching-shrinking nature of the carbon ink on an elastic PDMS substrate.

To investigate the repeatability of the sensor and reliability of the proposed fabrication approach, the DMA was used to cyclically stretch and release the sensor. In each cycle, the PDMS substrate was stretched to a maximum strain of $1 \%$ in $15 \mathrm{~s}$ and then released to its original state for another $15 \mathrm{~s}$ (Fig. 7). A maximum $\Delta R / R$ of $25.7 \%$ was achieved when the strain was increased to $1 \%$, while it fell to its base level after restored to the PDMS substrate. Figure 7 also shows the $\Delta R / R$ of the fabricated sensor for 30 cycles of stretching-releasing of the PDMS substrate, which shows a consistent $\Delta R / R$ change for the strain. Overall, the peak $\Delta R / R$ at a strain of $1 \%$ was always 

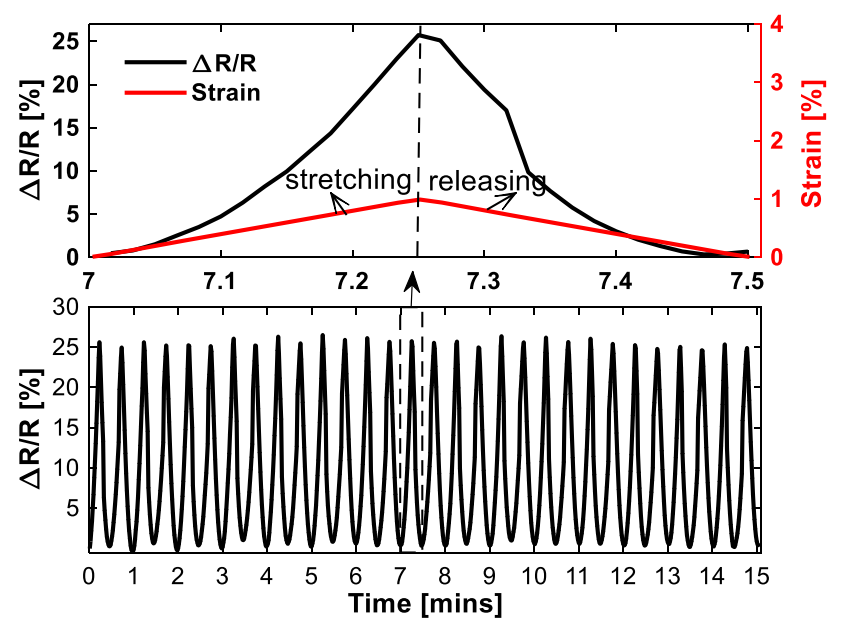

Figure $7 \Delta R / R$ of the sensor during the cyclic stretching and releasing tests for repeatability.

maintained at $25.5 \pm 0.5 \%$ over the cyclic operations. Finally, the repeatability test showed no cracks or delamination of the solidified carbon ink. This confirms the robust adhesion between the sensing material and the PDMS substrate in safe stretching limits.

\section{Conclusions}

In this work, we designed and fabricated a low-cost piezoresistive sensor by directly bonding solidified carbon ink film to a PDMS substrate. Mechanical, thermal, and electrical properties of the sensor were investigated. From the proposed fabrication procedure, the thickness of the sensor was about $45 \mu \mathrm{m}$, which is the thinnest reported so far. The temperature coefficient for the resistance was confirmed to be $0.37 \Omega /^{\circ}$, and the elasticity of the sensor was less at body temperature than at room temperature, all of which would be relevant for the design of a biomedical-related sensor. In the tensile experiments, the $\Delta R / R$ of the sensor increased linearly as a function of strain, with the maximum $\Delta R / R$ of about $122 \%$ being achieved when the strain reached $5 \%$, indicating the sensor's high sensitivity to external tension. The experimental results also demonstrate the stability, repeatability, and consistency of the $\Delta R /$ $R$ response to changes in strain, and the resistance increased with increasing strain, while it dropped to its original value when restored to the PDMS substrate. During the multiple stretching-releasing testing, peak $\Delta R / R$ was maintained at an average value of $25.5 \%$ for a strain of $1 \%$.

\section{Acknowledgements}

This work was made possible by NPRP grant NPRP11S-0104-180192 from the Qatar National Research Fund (a member of Qatar Foundation). The statements made herein are solely the responsibility of the authors.

\section{Funding}

Open Access funding provided by the Qatar National Library.

\section{Compliance with ethical standards}

Conflict of interest The authors declare that they have no conflict of interest.

Open Access This article is licensed under a Creative Commons Attribution 4.0 International License, which permits use, sharing, adaptation, distribution and reproduction in any medium or format, as long as you give appropriate credit to the original author(s) and the source, provide a link to the Creative Commons licence, and indicate if changes were made. The images or other third party material in this article are included in the article's Creative Commons licence, unless indicated otherwise in a credit line to the material. If material is not included in the article's Creative Commons licence and your intended use is not permitted by statutory regulation or exceeds the permitted use, you will need to obtain permission directly from the copyright holder. To view a copy of this licence, visit http://creativecommons.org/licen ses/by/4.0/.

\section{References}

[1] Muth JT, Vogt DM, Truby RL, Mengüç Y, Kolesky DB, Wood RJ, Lewis JA (2014) Embedded 3D printing of strain sensors within highly stretchable elastomers. Adv Mater 26:6307-6312

[2] Gong S, Schwalb W, Wang Y, Chen Y, Tang Y, Si J, Shirinzadeh B, Cheng W (2014) A wearable and highly sensitive 
pressure sensor with ultrathin gold nanowires. Nat Commun $5: 3132$

[3] Kim H-K, Lee S, Yun K-S (2011) Capacitive tactile sensor array for touch screen application. Sens Actuators A 165:2-7

[4] Fan F-R, Lin L, Zhu G, Wu W, Zhang R, Wang ZL (2012) Transparent triboelectric nanogenerators and self-powered pressure sensors based on micropatterned plastic films. Nano Lett 12:3109-3114

[5] Cheng M-Y, Huang X-H, Ma C-W, Yang Y-J (2009) A flexible capacitive tactile sensing array with floating electrodes. J Micromech Microeng 19:115001

[6] Zhao X, Hua Q, Yu R, Zhang Y, Pan C (2015) Flexible, stretchable and wearable multifunctional sensor array as artificial electronic skin for static and dynamic strain mapping. Adv Electron Mater 1:1500142

[7] Lipomi DJ, Vosgueritchian M, Tee BC-K, Hellstrom SL, Lee JA, Fox CH, Bao Z (2011) Skin-like pressure and strain sensors based on transparent elastic films of carbon nanotubes. Nat Nanotechnol 6:788-792

[8] Trung TQ, Lee N-E (2016) Flexible and stretchable physical sensor integrated platforms for wearable human-activity monitoring and personal healthcare. Adv Mater 28:4338-4372

[9] Yamamoto Y, Harada S, Yamamoto D, Honda W, Arie T, Akita S, Takei K (2016) Printed multifunctional flexible device with an integrated motion sensor for health care monitoring. Sci Adv 2:e1601473

[10] Wang X, Gu Y, Xiong Z, Cui Z, Zhang T (2013) Silkmolded flexible, ultrasensitive, and highly stable electronic skin for monitoring human physiological signals. Adv Mater 26:1336-1342

[11] Chen X, Shao J, An N, Li X, Tian H, Xu C, Ding Y (2015) Self-powered flexible pressure sensors with vertically wellaligned piezoelectric nanowire arrays for monitoring vital signs. J Mater Chem C 3:11806-11814

[12] Dagdeviren C, Su Y, Joe P, Yona R, Liu Y, Kim Y-S, Huang Y, Damadoran AR, Xia J, Martin LW et al (2014) Conformable amplified lead zirconate titanate sensors with enhanced piezoelectric response for cutaneous pressure monitoring. Nat Commun 5:4496

[13] Wang J, Jiu J, Nogi M, Sugahara T, Nagao S, Koga H, He P, Suganuma K (2015) A highly sensitive and flexible pressure sensor with electrodes and elastomeric interlayer containing silver nanowires. Nanoscale 7:2926-2932

[14] Chen Y-M, He S-M, Huang C-H, Huang C-C, Shih W-P, Chu C-L, Kong J, Li J, Su C-Y (2016) Ultra-large suspended graphene as a highly elastic membrane for capacitive pressure sensors. Nanoscale 8:3555-3564
[15] Yi Y, Wang B, Bermak A (2019) A low-cost strain gauge displacement sensor fabricated via shadow mask printing. Sensors 19:4713

[16] Wang Z, Wang S, Zeng J, Ren X, Chee AJY, Yiu BYS, Chung WC, Yang Y, Yu ACH, Roberts RC et al (2016) High sensitivity, wearable, piezoresistive pressure sensors based on irregular microhump structures and its applications in body motion sensing. Small 12:3827-3836

[17] Yi Y, Ali S, Wang B (2019) An inkjet-printed strain sensor with a carbon-silver polyimide topology. In: 2019 IEEE international conference on flexible and printable sensors and systems (FLEPS) 2019, pp 1-3

[18] Wang C, Hwang D, Yu Z, Takei K, Park J, Chen T, Ma B, Javey A (2013) User-interactive electronic skin for instantaneous pressure visualization. Nat Mater 12:899-904

[19] Berger C, Phillips R, Centeno A, Zurutuza A, Vijayaraghavan A (2017) Capacitive pressure sensing with suspended graphene-polymer heterostructure membranes. Nanoscale 9:17439-17449

[20] Cheng Y, Wang S, Wang R, Sun J, Gao L (2014) Copper nanowire based transparent conductive films with high stability and superior stretchability. J Mater Chem C 2:5309-5316

[21] Langley D, Giusti G, Mayousse C, Celle C, Bellet D, Simonato J-P (2013) Flexible transparent conductive materials based on silver nanowire networks: a review. Nanotechnology 24:452001

[22] Cohen DJ, Mitra D, Peterson K, Maharbiz MM (2012) A highly elastic, capacitive strain gauge based on percolating nanotube networks. Nano Lett 12:1821-1825

[23] Ryu S, Lee P, Chou JB, Xu R, Zhao R, Hart AJ, Kim S-G (2015) Extremely elastic wearable carbon nanotube fiber strain sensor for monitoring of human motion. ACS Nano 9:5929-5936

[24] Pang C, Lee G-Y, Kim T-I, Kim SM, Kim HN, Ahn S-H, Suh K-Y (2012) A flexible and highly sensitive strain-gauge sensor using reversible interlocking of nanofibres. Nat Mater 11:795-801

[25] Park M, Im J, Park J, Jeong U (2013) Micropatterned stretchable circuit and strain sensor fabricated by lithography on an electrospun nanofiber mat. ACS Appl Mater Interfaces $5: 8766-8771$

[26] Segev-Bar M, Landman A, Nir-Shapira M, Shuster G, Haick H (2013) Tunable touch sensor and combined sensing platform: toward nanoparticle-based electronic skin. ACS Appl Mater Interfaces 5:5531-5541

[27] Liu X, Zhu Y, Nomani MW, Wen X, Hsia T-Y, Koley G (2013) A highly sensitive pressure sensor using a Au-patterned polydimethylsiloxane membrane for biosensing applications. J Micromech Microeng 23:025022 
[28] Yao H-B, Ge J, Wang C-F, Wang X, Hu W, Zheng Z-J, Ni Y, Yu S-H (2013) A Flexible and highly pressure-sensitive graphene-polyurethane sponge based on fractured microstructure design. Adv Mater 25:6692-6698

[29] Chen Z, Ming T, Goulamaly MM, Yao H, Nezich D, Hempel M, Hofmann M, Kong J (2016) Enhancing the sensitivity of percolative graphene films for flexible and transparent pressure sensor arrays. Adv Func Mater 26:5061-5067

[30] Kong J-H, Jang N-S, Kim S-H, Kim J-M (2014) Simple and rapid micropatterning of conductive carbon composites and its application to elastic strain sensors. Carbon 77:199-207

[31] Wang L, Ding T, Wang P (2009) Thin flexible pressure sensor array based on carbon black/silicone rubber nanocomposite. IEEE Sens J 9:1130-1135

[32] Sepúlveda AT, Villoria RGD, Viana JC, Pontes AJ, Wardle BL, Rocha LA (2013) Full elastic constitutive relation of non-isotropic aligned-CNT/PDMS flexible nanocomposites. Nanoscale 5:4847-4854

[33] Shi J, Li X, Cheng H, Liu Z, Zhao L, Yang T, Dai Z, Cheng Z, Shi E, Yang L et al (2016) Graphene reinforced carbon nanotube networks for wearable strain sensors. Adv Func Mater 26:2078-2084

[34] Pang Yu, Tian He, Tao L, Li Y, Wang X, Deng N, Yang Yi, Ren T-L (2016) Flexible, highly sensitive, and wearable pressure and strain sensors with graphene porous network structure. ACS Appl Mater Interfaces 8(40):26458-26462

[35] Han J-W, Kim B, Li J, Meyyappan M (2013) Flexible, compressible, hydrophobic, floatable, and conductive carbon nanotube-polymer sponge. Appl Phys Lett 102:051903

[36] Song Y, Chen H, Su Z, Chen X, Miao L, Zhang J, Cheng X, Zhang H (2017) Highly compressible integrated supercapacitor-piezoresistance-sensor system with CNT-PDMS sponge for health monitoring. Small 13:1702091

[37] Liang B, Chen W, He Z, Yang R, Lin Z, Du H, Shang Y, Cao A, Tang Z, Gui X (2017) Highly sensitive, flexible MEMS based pressure sensor with photoresist insulation layer. Small 13:1702422

[38] Jian M, Xia K, Wang Q, Yin Z, Wang H, Wang C, Xie H, Zhang M, Zhang Y (2017) Flexible and highly sensitive pressure sensors based on bionic hierarchical structures. Adv Func Mater 27:1606066
[39] Yu G, Hu J, Tan J, Gao Y, Lu Y, Xuan F (2018) A wearable pressure sensor based on ultra-violet/ozone microstructured carbon nanotube/polydimethylsiloxane arrays for electronic skins. Nanotechnology 29:115502

[40] Zang Y, Zhang F, Di C-A, Zhu D (2015) Advances of flexible pressure sensors toward artificial intelligence and health care applications. Mater Horizons 2:140-156

[41] Yi Y, Buttner U, Carreno AAA, Conchouso D, Foulds IG (2015) A pulsed mode electrolytic drug delivery device. J Micromech Microeng 25:105011

[42] Yi Y, Buttner U, Foulds IG (2015) A cyclically actuated electrolytic drug delivery device. Lab Chip 15:3540-3548

[43] Ali S, Khan S, Bermak A (2019) Inkjet-printed human body temperature sensor for wearable electronics. IEEE Access 7:163981-163987

[44] Luheng W, Tianhuai D, Peng W (2007) Effects of conductive phase content on critical pressure of carbon black filled silicone rubber composite. Sens Actuators A 135:587-592

[45] Wang L, Cheng L (2014) Piezoresistive effect of a carbon nanotube silicone-matrix composite. Carbon 71:319-331

[46] Yamada T, Hayamizu Y, Yamamoto Y, Yomogida Y, IzadiNajafabadi A, Futaba DN, Hata K (2011) A stretchable carbon nanotube strain sensor for human-motion detection. Nat Nanotechnol 6:296-301

[47] Amjadi M, Pichitpajongkit A, Lee S, Ryu S, Park I (2014) Highly stretchable and sensitive strain sensor based on silver nanowire-elastomer nanocomposite. ACS Nano 8:5154-5163

[48] You B, Han CJ, Kim Y, Ju B-K, Kim J-W (2016) A wearable piezocapacitive pressure sensor with a single layer of silver nanowire-based elastomeric composite electrodes. J Mater Chem A 4:10435-10443

[49] Cui J, Zhang B, Duan J, Guo H, Tang J (2016) Flexible pressure sensor with Ag wrinkled electrodes based on PDMS substrate. Sensors 16:2131

Publisher's Note Springer Nature remains neutral with regard to jurisdictional claims in published maps and institutional affiliations. 